\title{
Publizistische Ethik und gesellschaftliche Realität
}

\author{
von Ulrich Saxer
}

Der 21. September 1943 brachte dem Columbia Broadcasting System einen seiner spektakulärsten Erfolge, dem Unterhaltungsstar Kate Smith einen überwältigenden persönlichen Triumph, dank beiden den Hütern der amerikanischen Währung die Zeichnung von Kriegsanleihen im Werte von 39 Millionen Dollar und der Massenkommunikationsforschung eine ihrer bedeutendsten Untersuchungen'. Deren Verfasser indes, Robert K. Merton, schließt seinen Forschungsbericht zu diesem Tag, der doch so augenscheinlich die Macht der Massenmedien, das Vernünftige zu erwirken, bewiesen hat, mit dem Kapitel: „Mass Persuasion: A Technical Problem and a Moral Dilemma"2 und zweifelt darin sogar auch am ethischen Gehalt der von ihm selbst mit Meisterschaft praktizierten Sozialwissenschaft ${ }^{3}$.

Unter deren geheimer Assistenz hatte die beliebte Unterhaltungssängerin in einem sorgfältigst geplanten 18stündigen Radiomarathon eine solche Anzahl von Hörern aus ihrem Massenpublikum zum Erwerb dieser Obligationen veranlassen können, daß schließlich diese gewaltige Summe zustandekam: für die Käufer eine ansprechend verzinste Investition, für die nationale Währung eine gewisse Sicherung gegen künftige inflatorische Aushöhlung. Freilich verdankte sich Kate Smiths Überredungserfolg gerade nicht der Erläuterung dieser wirtschaftlichen Zusammenhänge und Vorteile, sondern vielmehr ihrer systematischen Verschleierung durch virtuos gestaffelte Appelle an eine ganz andere patriotische Opferbereitschaft, an geheime Angste und Identifikationsbedürfnisse aller Art, und dies war es hauptsächlich, was Mertons Argwohn erregte, hier habe weniger das Gute die Unvernunft als die publizistische Technik die publizistische Ethik besiegt.

Solcher Zweifel am Ethos der Publizierenden ist ebenso alt wie unstillbar und hat sich denn auch vor und nach dem 21. September 1943 vielerorts in immer neuen Forderungen nach publizistischen Verhaltenskodizes und Kontrollinstitutionen, aber auch in gesetzlichen Regelungsversuchen und natïrlich in unablässiger Kritik niedergeschlagen. Alt ist allerdings auch jene Ratlosigkeit, die selbst Mertons Anmerkungen kennzeichnet und die trotz vieler gegenteiliger Beteuerungen zu dem Schluß zwingt, im Problem der publizistischen Ethik spiegelten sich gesamtgesellschaftliche Widersprüche, denen nicht einfach durch moralische Forderungen an den Berufsstand der Publizisten beizukommen sei.

Im Folgenden soll denn auch keine weitere "Ethik des Informierens" ${ }^{{ }_{4}}$ oder gar des Publizierens schlechthin entworfen und postuliert werden, sondern es sollen bloß dimensionsanalytisch einige gesellschaftliche Bedingungskonstellationen aufgezeigt werden, unter denen den Publizisten die Verwirklichung ethischer Werte angesonnen wird. Mit dieser Beschränkung auf das Vorfeld der eigentlichen ethischen Diskussion, d. h. mit dem Verzicht auf die Abwägung des je zu vertretenden Guten, bezeugen also auch diese Ausführungen jene Verlegenheit der sich zur Werturteilsabstinenz verpflichtenden Sozialwissenschaft, auf die Merton anspielt ${ }^{5}$.

Dr. Ulrich Saxer ist Mitarbeiter der „Neuen Zürcher Zeitung“ und freier wissenschaftlicher Mitarbeiter des Journalistischen Seminars der Universität Zürich. 
Trotzdem dürfte eine solche bloße Vorarbeit im Problembereich der publizistischen Ethik aus zwei Gründen nicht ganz nutzlos sein: 1. als empirische Korrektur der einseitig idealistisch-ideologischen Diskussion zur publizistischen Ethik und 2. als Würdigung der publizistischen Rollenproblematik in einem weiteren gesellschaftlichen Kontext, wie sie zur Widerlegung sachfremder Kritik auch von publizistischer Seite immer wieder gefordert wird. Es werden hier also sowohl die Publizistik als auch die Bemühungen um deren ethische Standards wie schon von Merton in einen gesamtgesellschaftlichen Zusammenhang gebracht. Nur mit diesem weiten Ansatz läßt sich nämlich einesteils Massenkommunikation als ein soziales Totalphänomen $^{6}$, das sich in den verschiedenen Lebensbereichen ausprägt, adäquat erfassen und andernteils die Frage nach der publizistischen Ethik einigermaßen ideologiefrei, vor allem ohne die Projektion anderswo lokalisierter ethischer Problematik auf die Berufsgruppe der Publizisten, beantworten.

Den zwei erwähnten Hauptzielen dieser Untersuchung gemäß sollen im übrigen zuerst die wichtigsten soziokulturellen Dimensionen des Postulats einer publizistischen Ethik, gewissermaßen sein gesellschaftlicher Hintergrund, erläutert und hierauf, zweitens, die objektiven Möglichkeiten und Schwierigkeiten, ethische Werte in der publizistischen Arbeit zu verwirklichen, umrissen werden. Als methodologisches Hauptinstrument wird dabei der sogenannte Idealtypus eingesetzt. Darunter ist im Unterschied zum Real- oder Durchschnittstyp eine theoretische Hilfskonstruktion zu verstehen, die zwar von der Fülle der realen Daten und Beziehungen ausgeht, diese aber zwecks größerer Deutlichkeit nicht nur auf das Wesentliche reduziert, sondern das an ihnen theoretisch Bedeutsame auch überprofiliert. Idealtypen sind also mit anderen Worten vorläufige Theoretisierungsversuche und Orientierungshilfen bzw. Veranschaulichungsmittel zugleich?

\section{Publizistische Ethik als Postulat}

Zwei Eigentümlichkeiten der Diskussion um die publizistische Ethik legen es vor allem nahe, diesen Problemkomplex im Lichte gesamtgesellschaftlicher Gegebenheiten zu erörtern. Eine besondere publizistische Ethik wird ja, erstens, durchaus nicht von jedermann und universell gefordert, sondern vornehmlich in demokratischen Industriegesellschaften. Es ist (1.) überhaupt einmal die Legitimität dieses Postulats zu untersuchen. Zweitens fällt auf, daß von den verschiedenen gesellschaftlichen Kräften mit sehr unterschiedlicher Intensität ethische Vorschriften bezüglich der Publizisten entworfen und oktroyiert werden. Es müssen folglich (2.) die Eigenart und die Ziele wenigstens von zwei verschiedenen postulierenden Gruppen analysiert werden.

\section{Die Legitimität des Postulats}

Die Forderung nach einer zusätzlichen oder besonderen publizistischen Ethik ist offenbar dann gerechtfertigt, wenn die bestehende gesellschaftliche Regelung der Massenkommunikation keine befriedigende Erfüllung der für die Gesellschaft unentbehrlichen Leistungen der Massenmedien sichert. Ohne jegliche gesellschaftliche Regelung vollzieht sich nämlich Massenkommunikation auf keinen Fall, sind doch die Massenmedien als soziokulturelle Institutionen ausgestaltet und erfüllen mithin die ihnen übertragenen Funktionen der Umweltüberwachung, der Meinungskorrelation, der Kulturtransmission und der psychischen Gratifikation immer gemäß 
einer gewissen gesellschaftlichen Normierung. Die entscheidende erste Frage ist also, unter welchen Umständen diese Funktionserfüllung trotzdem als unbefriedigend qualifiziert werden kann.

Das Beispiel von Kate Smiths Sendung kann hier weiterhelfen. Ihre Radioappelle mobilisierten ein Massenpublikum zu einem Verhalten, das sowohl seinen Interessen als auch dem wirtschaftlichen Gemeinwohl dienlich war, und aktualisierten erst noch die nationale Wertwelt in ihren Zuhörern. Zur Erreichung dieses nationalen Solidarisierungserfolgs und damit zur Verwirklichung bestimmter anerkannter, wichtiger ethischer Postulate verletzten indes die Publizisten andere kulturelle Normen, namentlich die Verpflichtung zu einer für den Bestand der geltenden politischen Ordnung wichtigen Informationswahrheit, deren kulturspezifisches Erfordernis als

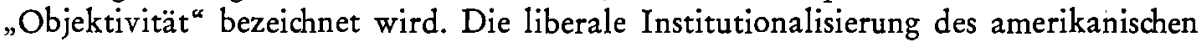
Hörfunks gestattete hier somit eine zwar wirkungsmächtige, aber nur teilweise normkonforme publizistische Aktion. Die von Merton angedeutete Forderung nach einer besseren publizistischen Ethik drückt folglich im Grunde genommen den Wunsch aus, Widersprüche zwischen der gesellschaftlichen Wertwelt und dem tatsächlichen gesellschaftlichen Funktionieren bzw. solche innerhalb des Systems kultureller Normen sollten ausgeglichen werden.

Die Frage, unter welchen Umständen die Leistungen der Massenmedien als nicht befriedigend gelten könnten, erweist sich damit - durchaus nicht erstaunlicherweise - als zu wenig differenziert. Die Funktionsfülle der Massenmedien und die Heterogenität ihrer Publica lassen ja kaum eine generelle Antwort zu. Einer Mehrheit von Zuhörern Kate Smiths, die von der Darstellungsqualität wie vom gesellschaftlichen Nutzen der Sendung überzeugt war, stand beispielsweise eine Minderheit, darunter Merton, gegenüber, die den Wert dieser publizistischen Produktion bezweifelte. Die Sängerin und ihre publizistischen Ratgeber wiederum entschieden sich einfach in einem gesellschaftlich bzw. institutionell gegebenen Normenkonflikt unter ziemlich allgemeinem Beifall für eine bestimmte Lösung, die indes jener Minderheit unannehmbar und Ausdruck mangelnder publizistischer Ethik schien. Das Problem, wie legitim das Postulat einer besonderen publizistischen Ethik sei, führt mithin unweigerlich zur Frage nach der Instanz, welche überhaupt über die Qualität der Medien zu befinden und eventuell besondere ethische Richtlinien zu erlassen habe.

Damit rückt erneut die Gesamtgesellschaft ins Blickfeld, welche offenbar die Massenmedien unzulänglich institutionalisiert. Der Vergleich zwischen totalitären und fundamental-demokratischen Systemen ergibt hier, daß in den ersteren zwar leicht eine solche Instanz zu bezeichnen wäre, das ganze Problem aber von geringer Relevanz für das System ist, während in den letzteren kaum eine solche Instanz ausgemacht werden kann, der Frage der publizistischen Ethik indes eine ganz erhebliche Bedeutung für das System zukommt. Die Zwangsverstaatlichung der Gesellschaft im ersten Fall bedeutet ja stets auch eine minutiöse Regelung der publizistischen Aktivität, und zwar in zwingendster Form: durch staatliche Setzungen. Der Spielraum ethischen Ermessens für die Publizisten, die überdies normalerweise dank ihrer bewiesenen Loyalität zur herrschenden Machtelite ihre Funktionen ausüben, ist dadurch dermaßen beschnitten, daß sich seine Diskussion hier erübrigt. Dementsprechend eindeutig ist es an der Machtelite, die ja z. B. auch schon die kulturellen Normen soweit möglich nach ihren politischen Bedürfnissen hierarchisiert hat, über die Qualität des Veröffentlichten zu befinden 8 .

Die zwangsverstaatlichte Gesellschaft ist also im idealtypischen Fall durch ein relativ widerspruchsfreies Wertsystem, dessen Homogenität freilich erzwungen wird, 
eine endeutig designierte Instanz, welche die Massenmedien qualifiziert und eine lückenlose, absolut verbindliche gesellschaftliche Regelung der Massenkommunikation charakterisiert. Die demokratische Gesellschaft hingegen, wie Kate Smiths Sendung beweist, erfüllt kein einziges dieser drei Merkmale. In ihr gilt ja einmal für die Aktivität der Massenmedien das Prinzip der Pressefreiheit. Verwirklicht wird dieses idealtypisch auf zwei Arten: Entweder konkurrieren die Massenkommunikationsmittel im Rahmen der allgemeinen staatlichen Gesetzgebung als Wirtschaftsunternehmen auf dem Kommunikationsmarkt oder sie produzieren als Monopolbetriebe unter halb staatlicher, halb gesellschaftlicher Kontrolle gemäß demokratischen Proportionalitätsprinzipien. Entscheidend ist, daß in beiden Fällen den publizistischen Institutionen eine gewisse Automonie gegenüber der Staatsmacht und ihrer Gesetzgebung eingeräumt wird, ja daß sogar gesetzliche Privilegien ihnen eine besonders ungehinderte Tätigkeit garantieren.

Der Zweck, den die demokratische Gesellschaft mit dieser Befreiung der Massenmedien von staatlichem $Z$ wang und damit von der Verpflichtung auf eine bestimmte publizistische Linie verfolgt, ist natïrlich die unbehinderte Zirkulation der Informationen. Publizistik im totalitären Staat ist in erster Linie prägend, ein Instrument, das von der Machtelite auf die Gesellschaft angesetzt wird; in der Demokratie hingegen ist sie spiegelnd, Ausdruck und Selbstdarstellung der demokratischen Gesellschaft. Schon der Verzicht selber, die Massenmedien durchgehend staatlich zu normieren, zeugt von diesem kollektiven Selbstverständnis, die Gesellschaft gehe nicht im Staat auf und daher müßten auch ihre ganz unterschiedlichen Regulierungsmechanismen die publizistischen Prozesse mitregeln. Weitere allgemeine und spezielle kulturelle Normen, darunter ästhetische und eben auch ethische, bestimmen mithin stets die Publizistik der demokratischen Gesellschaft. Ihren Publizisten gewährt sie damit ganz beträchtliche Wahlmöglichkeiten zwischen verschiedenen Zwecken und Mitteln.

Umgekehrt setzt aber dieser freiheitliche Institutionalisierungsmodus der Massenmedien ganz selbstverständlich voraus, daß sich die Publizisten nicht nur durch die staatliche Gesetzgebung gebunden fühlen. Diese spart sie ja gerade aus, privilegiert sie sogar vielfach, weil ihre Tätigkeir im öffentlichen Interesse liege. Beides geschieht natürlich in der Annahme, die Publizisten hielten sich zum ersten an die gesellschaftlich gängige Moral, und sie rechtfertigten, zweitens, ihre Privilegierung durch eine entsprechende berufliche Spezialethik. Ethische Verpflichtungen der Publizisten sind also mit der demokratischen Institutionalisierung der Massenkommunikationsmittel zum vornherein mitgesetzt, die Forderung, sie möchten diesen nachkommen, mithin legitim ${ }^{9}$.

Das Postulat einer publizistischen Ethik, die Dauerdiskussion darum in den modernen demokratischen Gesellschaften, verraten sogar an sich ein verbreitetes Verständnis für die systemgemäßen Erfordernisse der publizistischen Tätigkeit. Eine befriedigende Verwirklichung dieser Forderungen hängt allerdings, wie angedeutet, an der Möglichkeit, verbindliche Beurteilungsinstanzen für die Qualität der Publizistik auszumachen, und an der Einheitlichkeit des jeweiligen gesellschaftlichen Normensystems, und beide Bedingungen sind eben kaum je erfüllt.

\section{Postulierende Gruppen}

Die verschiedenen Gruppen, die eine bessere publizistische Ethik verlangen, tun dies ja regelmäßig weder bei denselben Anlässen noch im gleichen Sinne, sehr oft indes 
mit den gleichen Argumenten und Begriffen. Idealtypisch sind hier in erster Linie politische, bzw. wirtschaftliche Kräfte, z. B. Parteien und Verbände, und kulturrepräsentierende und -vermittelnde Kreise, z. B. kirchliche und pädagogische, von Bedeutung. Politische, wirtschaftliche und kulturelle Werte, Bedürfnisse und Interessen dedken sich also keineswegs, polarisieren sich vielmehr gerade bei dieser Diskussion häufig, und zugleich offenbart sich hier eine starke Spannung $z$ wischen gesellschaftlicher Wertwelt und tatsächlichem gesellschaftlichem Funktionieren überhaupt. Dieser Zustand mehr oder minder gravierender gesellschaftlicher Anomie ${ }^{10}$ zeigt sich dabei vor allem eben auch im Gegensatz zwischen jenen Instanzen, die politische und wirtschaftliche Projekte verwirklichen, und jenen, die vornehmlich kulturelle Normen artikulieren, tradieren und überwachen.

Die publizistischen Institutionen, als soziokulturelle, sind natürlich diesen Widersprüchen in besonderem Maß ausgesetzt. Seien sie marktabhängig oder halbstaatlich, stets haben ihre Leistungen dem tatsächlichen gesellschaftlichen Funktionieren zu dienen und doch den kulturellen Normen zu genïgen, da ja bloß unter dieser Voraussetzung auf ihre lückenlos $z$ wingende gesetzliche Regelung verzichtet wird. Kate Smith z. B. befriedigte mit ihren Radioappellen höchst erfolgreich verbreitete sozialpsychische Bedürfnisse und förderte auch das wirtschaftliche Gemeinwohl, indes mit einer anfechtbaren publizistischen Technik. Diese verstieß nämlich gegen die politisch wie gesamtkulturell ebenfalls gegebene Verpflichtung der Publizisten, den Rezipienten durch objektive Urteilsgrundlagen sachangemessene individuelle Entscheidungen zu ermöglichen.

Die für die Sendung Verantwortlichen konnten dabei allerdings die kollektive Notsituation, den Kriegszustand, geltend machen, der zur Währungssicherung auch problematische Mittel rechtfertige, um so mehr als ihre Aktion auch der nationalen Wertintegration gedient habe. Gesinnungsethisch sei ihr Verhalten zwar kritisierbar, verantwortungsethisch aber weitgehend vertretbar. Dem kann entgegengehalten werden, bei diesem manipulativen Einsatz des Massenkommunikationsmittels seien nicht nur die Würde der rezipierenden Individuen und die kulturelle Wahrheitsnorm verletzt worden; sondern auch die Basis des demokratischen Konsenses, ein gewisses Vertrauen, werde auf diese Weise letztlich unterhöhlt; nicht nur prinzipiell, sondern auch seiner tatsächlichen Folgen wegen sei dieses publizistische Wirken verwerflich. $\mathrm{Zu}$ den Widersprüchen zwischen den gesamthaft zu respektierenden Normen kommen mithin auch die Unterschiede $z$ wischen den jeweiligen Situationen, in denen publiziert wird, und dies in einem gesellschaftlichen Ganzen, das durch unterschiedliche und häufig schwache Internalisierung vieler kultureller Normen charakterisiert ist. Die Postulate der verschiedenen Gruppen an die Publizisten müßten also für diese die widerstrebenden Normen entweder prinzipiell und verbindlich hierarchisieren oder zumindest verpflichtende situationsethische Lösungen darstellen. Dabei müßten insbesondere gesinnungs- und verantwortungsethische Positionen versöhnt werden können, deren von Max Weber beschriebener Gegensatz offenbar auch noch in den westlichen Gesellschaften von heute Ausdruck elementarer Norm- und Rollenkonflikte ist ${ }^{11}$.

Die Vorschläge der postulierenden Gruppen fallen indes in Wirklichkeit regelmäßig dermaßen positionsgebunden aus, daß an ihnen das Fehlen einer allgemein anerkennbaren Qualifikationsinstanz für publizistische Leistungen nur zu offenkundig wird. Idealtypisch reagieren dabei z. B. die erwähnten politischen und wirtschaftlichen Kreise gewöhnlich okkasionell, d.h. immer dann, wenn Publizistik ihnen ihrer Meinung nach zu Unrecht die Durchsetzung ihrer Projekte erschwert. Die 
Forderung nach einer besseren publizistischen Ethik wird dabei von ihnen hauptsächlich im Namen eines störungsfreien gesellschaftlichen Funktionierens und der demokratischen Wertwelt erhoben. Da aber diese erst in der Gesetzgebung ihre wirklich verpflichtende Konkretisierung erhält und die Publizistik davon ja gerade weitgehend ausgespart bleibt, wird damit im Grunde nur ein Vorrang des Politischen oder Wirtschaftlichen vor dem Kulturellen behauptet, mangels zwingenderer Mittel aber zugleich auf kulturelle Normen rekurriert und aus der widersprüchlichen Fülle derselben Bestimmtes für das wirklich Verbindliche erklärt.

Der Bereich der nichtgesetzlichen kulturellen Normierung, aus dem also die Postulate einer publizistischen Ethik abgeleitet werden müssen, erweist sich somit in solchen Gesellschaften als recht beliebig beanspruchbar. Dies ist vor allem ein Resultat des für sie charakteristischen kulturellen Pluralismus, der ein widersprüchliches und gerade von den Massenmedien allenthalben vermitteltes Überangebot an kulturellen Normen zeitigt, viele davon bloß partiell oder überhaupt kaum mehr internalisiert. Dieser kulturelle Pluralismus selber ist wieder Ausdruck einer Gesellschaft, die durch die Beschränkung der staatlichen $Z$ wangsgewalt kulturelle Vielfalt gewähren lassen will12; daß die Massenmedien diese spiegeln, ist daher durchaus systemgerecht. Zugleich vollzieht sich aber damit das tatsächliche gesellschaftliche Funktionieren einesteils zwar gemäß recht verschiedenartigen kulturellen Standards, andernteils aber auch unter einer Art von kulturellem, insbesondere auch moralischem, Uberbau, der das individuelle und gruppenhafte Verhalten nur sehr bedingt und gesamthaft, jedenfalls schwach steuert. Dafür steht mit ihm den in diesen komplexen Gesellschaften tätigen Symbol- und Kommunikationsspezialisten ein Arsenal von kulturellen, darunter auch ethischen, Legitimationen zur Verfügung, unter denen diese in der hauptsächlich durch Massenmedien hergestellten Offentlichkeit bestimmte partikuläre Ansprüche und Interessen vertreten. Das Korrelat des kulturellen Pluralismus bildet hier mit andern Worten eine massive Ideologisierung kultureller Normen, mit dem Resultat zusätzlicher Orientierungsschwierigkeiten für Gutgläubige bzw. der Abwertung artikulierter kultureller Verbindlichkeiten überhaupt.

Die Postulate der als Kulturrepräsentanten bezeichneten Kreise sind unter diesen Umständen materiell natürlich auch verschieden, ihre Argumentationsweise dafür gesamthaft umso ähnlicher. Im Unterschied zu den politischen und wirtschaftlichen Kräften fordern sie z. B. weniger okkasionell denn prinzipiell, andauernd, und zwar regelmäßig im Namen "wahrer" oder „echter" Kultur. Wenn die Selektion und Präsentation des publizierten Materials den Standards dieser jeweiligen Kulturideale entspricht oder diese gar ausdrücklich vertritt, dann erfüllen unter dieser Optik die Publizisten die ethische Norm.

Damit spiegeln allerdings die Forderungen der kulturrepräsentierenden und -vermittelnden Kreise im allgemeinen auch bloß wieder deren eigene gesellschaftliche Position. Gewiß versehen sie in der gesellschaftlichen Arbeitsteilung Aufgaben der Artikulierung, Tradierung und bis zu einem gewissen Grad auch Sanktionierung kultureller Normen und Muster, die Publizisten aber ja nicht minder. Die Kritik an der publizistischen Ethik, die etwa von Kirchen und Schulen ausgeht, ist also maßgeblich auch ein Kontrollversuch durch konkurrierende Institutionen, während die politischen und wirtschaftlichen Mächte damit in erster Linie ihren Willen als Benützer der Massenmedien kundtun. Die typische Leistung von Kirchen und Schulen ist dabei die Systematisierung kultureller Muster und Normen, diejenige von Publizistik im gesamten hingegen, ihrem Institutionalisierungssinn nach, bloß die Repräsentation der kulturellen Vielfalt. 
Die prinzipielle Forderungsweise der ersteren wird daraus noch einmal verständlich, freilich auch deren beschränkte Sachgerechtigkeit und Gültigkeit. Den aus solchen pädagogischen und moralischen Systematiken abgeleiteten publizistischen Verhaltensregeln eignet ja auch wieder bloß eine ähnlich partikuläre Verbindlichkeit wie den ad-hoc-Forderungen der politischen und wirtschaftlichen Kräfte, und situationsethische Entscheidungshilfe im Widerspruch der Normen vermögen sie ihrer meist abstrakt-prinzipiellen Fassung wegen den Publizisten auch kaum zu geben. Der kulturelle Pluralismus solcher Gesellschaften zeitigt eben lediglich die Konkurrenz solcher kulturrepräsentierender und -vermittelnder Institutionen und keine ihn überwindende Instanz. Die dauernden Anstrengungen der andern kulturrepräsentierenden und -vermittelnden Gruppen, die Publizisten auf ihre bestimmten sittlichen Normen und Kulturstandards und nur diese zu verpflichten, sind darum gesamthaft auch nicht erfolgreicher als die okkasionellen Versuche der politischen und wirtschaftlichen Kräfte. Wie jene vermögen sie nur gemäß dem tatsächlichen gesellschaftlichen Einflußgefälle die publizistische Tätigkeit ethisch zu normieren.

In den modernen demokratischen Gesellschaften bemühen sich also verschiedene Gruppen, aber eben bloß als selbsternannte Instanzen, die publizistische Ethik zu verbessern. Ihre Postulate sind gewöhnlich Ausdruck ihrer eigenen Position und folglich in erster Linie der Versuch von Gruppen, den größtmöglichen Teil der Gesamtpublizistik mit ethischen Mitteln zu kontrollieren. Was sie damit über den Bereich der ohnehin von ihnen abhängigen Publizistik erreichen, ist freilich meist bloß eine gewisse allgemeine Zustimmung zu ethischen Allgemeinformeln, die aber von den Publizisten in den täglichen Arbeitssituationen dann doch nach individuellem Gutdünken und gemäß dem tatsächlichen Einflußgefälle ausgelegt werden. Der Positionsgebundenheit der von verschiedenen Gruppen geforderten publizistischen Ethiken entspricht indes diese bloß partielle Verbindlichkeit; erst über die staatliche Gesetzgebung werden sie für alle Publizisten verpflichtend. An Versuchen der postulierenden Gruppen, ihre Art von publizistischer Ethik zum Gesetz für alle zu machen, mangelt es denn auch nicht, selbstverständlich immer um des Gemeinwohls willen.

Dies bedeutet indes, daß eine Majorität des Volkes bzw. der Parlamentarier für eine solche Beschränkung des Prinzips der Pressefreiheit gewonnen werden muß. Die Instanz, die in solchen Gesellschaften über die Qualität der Publizistik gültig befinden und eine entsprechende Ethik tatsächlich sanktionieren kann, ist mit andern Worten eine immer wieder wechselnde gesamtgesellschaftliche Mehrheit und darum nicht fest designierbar. Sie bestimmt, auch wieder bloß von Fall zu Fall, was "Gemeinwohl“ materiell heißen soll. Eine Mehrheit findet sich aber bezeichnenderweise in diesen Gesellschaften gewöhnlich nicht zu mehr als zur zwingenden Durchsetzung allgemeiner kultureller Selbstverständlichkeiten in der Publizistik bereit, weil sie die Verschiedenartigkeit der publizistischen Produktion als Funktionserfordernis und adäquaten Spiegel solcher Gesellschaften erachtet.

Die Publizisten bleiben damit als unentbehrliche Spezialisten von einer schlechthin zwingenden Mehrheitskontrolle weitgehend verschont ${ }^{13}$, aus der Einsicht heraus, daß sie nur so die publizistische Selbstdarstellung der demokratischen pluralistischen Gesellschaft leisten können. Den verschiedenen gesellschaftlichen Einflüssen werden sie folglich preisgegeben, und doch wird ein besonderes ethisches Verhalten von ihnen erwartet, das indes von keiner klaren Instanz vorartikuliert, überwacht, sanktioniert wird. Zwar treten verschiedene postulierende Gruppen mit diesem Anspruch auf, aber ihre Forderungen sind letztlich Ausdruck „falschen“ Bewußtseins 
oder der Versuch, solches zu verbreiten. Die demokratischen modernen Gesellschaften rechnen also mit einer besondern Berufsethik der Publizisten als Gesamtgruppe und behindern durch ihre soziokulturelle Realität zugleich die Kodifizierung und Praktizierung einer solchen.

\section{Publizistische Ethik als Realität}

Trotzdem liegen in solchen Gesellschaften berufsethische Codices für die in Presse und Film, an Radio und Fernsehen Tätigen in großer Zahl vor ${ }^{14}$, Ethiken, die sich gesamthaft erst noch stark gleichen. Das legitime Postulat einer besonderen publizistischen Ethik bringt also zumindest entsprechende Wegleitungen hervor, deren Inhalt und Verbindlichkeit nach dem Vorherigen freilich problematisch sein dürfte. Es sind daher (1.) diese publizistischen Codices im Lichte der beschriebenen gesamtgesellschaftlichen Wirklichkeit zu würdigen. Dabei steht zum vornherein fest, daß solche Richtlinien zwar möglicherweise den Publizisten bei ihren Entscheidungen in Normkonflikten helfen können, diese aber nicht etwa aus der Welt schaffen. Es sollen deshalb (2.) noch solche grundsätzlichen publizistischen Rollenkonflikte zur Sprache kommen.

\section{Publizistische Codices}

Unter diesen zusätzlichen Regelungsversuchen von Publizistik sind idealtypisch diejenigen, die auf publizistische Institutionen zielen, von denjenigen zu unterscheiden, welche die Publizisten selber betreffen. Natürlich stellen diese definitionsgemäß einen sehr wichtigen Teil des Personals, durch das die publizistischen Institutionen überhaupt ihre Funktionen erfüllen; weil aber eben Personal und Institutionen hier wie überall keineswegs identisch sind, bestehen auch prinzipielle Unterschiede zwischen den beiden Arten von publizistischen Codices.

Immerhin nicht solch radikale, daß sie die bedeutenden Gemeinsamkeiten dieser Codices verdunkelten. Deren wichtigste ist, daß im Grunde alle jenen Qualifikationsmaßstab bzw. jene Qualifikationsinstanz implizieren, deren Fehlen im Vorherigen begründet wurde. Regelmäßig wird nämlich in ihnen die besondere Verantwortung der Publizisten und Massenmedien gegenüber der Offentlichkeit bzw. der Gesellschaft hervorgehoben und die Qualität solch verantwortungsvoller Publizistik am Gemeinwohl und an der kulturellen Wertwelt gemessen. $\mathrm{Da}$ aber das erstere nur von Fall zu Fall bestimmt werden kann und die letztere widersprüchlich ist, enthalten diese prinzipiellen Partien der publizistischen Codices gewöhnlich auch nicht viel mehr als jene kulturellen Selbstverständlichkeiten, die schon das Gesetz vorschreibt, und, gewiß, eine Fülle von ethischen Allgemeinformeln ${ }^{15}$, die indes fast jeder Auslegung zugänglich, erst in situationsethischen Konkretisationen hilfreich werden und nur bei entschiedener Sanktionsgewalt und -bereitschaft die publizistische Berufsausübung tatsächlich steuern.

Gerade ihrer Allgemeinheit wegen sind freilich diese ethischen Formeln vielfach die gleichen wie diejenigen, welche die postulierenden Gruppen vertreten und entstammen auch meist dem gleichen, früher erwähnten Arsenal. Ein Einverständnis zwischen diesen Gruppen und den Publizisten, die ja im Normalfall diese Codices als Verbandsarbeit selber verfassen und genehmigen, besteht aber gerade nicht darum postulieren schließlich jene. Die Ưbereinstimmung ist also bloß eine verbale, und das dürfte häufig auch der Sinn dieser allgemeinen Fundierungs- und Umschreibungsversuche einer publizistischen Ethik in diesen Codices sein. Was als 
materiale Bestimmung der eigenen berufsethischen Basis beliebig dürftig anmutet, dient also wohl in Wahrheit vor allem der Beschwichtigung der postulierenden Gruppen.

Unter den Kodifikationen, welche sich auf publizistische Institutionen beziehen, sind natürlich wieder diejenigen für rein privat kontrollierte Massenmedien von denjenigen für halbstaatlich institutionalisierte verschieden, freilich auch wieder nicht dermaßen, daß nicht auch bestimmte Ahnlichkeiten sofort auffielen. Da ja in den allgemeinen Klauseln eine positive ethische Fundierung der publizistischen Tätigkeit nicht recht glückt, wird hier nämlich regelmäßig versucht, sozusagen negativ: durch die Bezeichnung bestimmter publizistischer Schonbezirke, zu einer publizistischen Ethik zu kommen. Diese Schonbezirke sind im wesentlichen immer die gleichen: einerseits die Jugendlichen, andererseits Religion, Ehe und Familie, gewisse kulturelle Institutionen also $^{16}$.

Die vorher erst vermutete Konzession an postulierende Gruppen wird hier offenkundig, decken sich doch diese Schutzsphären recht genau mit den Bereichen, die z. B. Schulen und Kirchen am intensivsten zu kontrollieren suchen. Die von publizistischer Seite verfaßten und veröffentlichten Ethik-Entwürfe erweisen sich damit erwartungsgemäß viel stärker als Zeugnisse von Strategie denn von Philosophie, als Resultate des gesellschaftlichen Einflußgefälles denn des ethisch bemühten Sichtens von Normwidersprüchen. Im Grunde rät diese negative publizistische Ethik den Publizisten einfach zur Vorsicht in Bereichen, wo die konkurrierenden Institutionen wahrscheinlich sanktionsmächtig genug sind, die publizistische Unterbietung ihrer kulturellen Standards empfindlich zu ahnden.

Die kodifizierte Ethik der publizistischen Institutionen verpflichtet also diese dazu, gewisse Publica und Märkte nur mit Produkten eines bestimmten kulturellen Standards zu beliefern, der im kulturellen Pluralismus dieser Gesellschaft keineswegs allgemein verbindlich ist. Diese ergiebigen Märkte gerade mit einem andern Angebot als die konkurrierenden Institutionen zu erobern, ist indes ein Erfolgserfordernis von Publizistik, die publizistische Erfassung möglichst vieler Bevölkerungsschichten in solchen komplexen Großgesellschaften eine Notwendigkeit, die publizistische Repräsentation verschiedener kultureller Standards legitim. So erstaunt es denn nicht, $\mathrm{da} ß$ in den konkreteren Weisungen solcher Codices die publizistische Realitär immer wieder diese fremdbestimmte und darum negative publizistische Moral durchlöchert, ganz zu schweigen von der tatsächlichen Produktion. In den peniblen Katalogen tolerierter $\mathrm{Küsse}$, Morde und Entblößungen ${ }^{17}$ zumal zeigen sich mit ebenso lächerlicher wie betrüblicher Drastik der Widerspruch zwischen angenommener ethischer Instanz und gängiger Praxis und zugleich ein Schematismus, der weder der Komplexheit und der Verschiedenartigkeit der publizistischen Arbeitssituationen noch der Vielfalt der publizistischen Gestaltungsmöglichkeiten Rechnung trägt. Daß man von diesen primitiven Normierungsversuchen, die zur publizistischen Doppelmoral geradezu einladen, allmählich abkommt und vermehrt durch situationsethische Kasuistik anhand von konkreten, realen Fällen ethische Klärung und Steuerung anstrebt ${ }^{18}$, bedeutet da immerhin einen beträchtlichen Fortschritt. Dennoch dürfte eine Publizistik, die einer bestimmten elitären oder traditionalistischen Kulturideologie Lippendienste leistet und sie gleichzeitig mit ihrer Präsentationstechnik doch ständig verrät, Zynismus bei Publizisten und Rezipienten zeitigen.

Freilich können auch diese kodifizierten Kulturverpflichtungen erst im Lichte ihrer tatsächlichen Sanktionierbarkeit richtig gewürdigt werden. Den Codices, die halbstaatliche Institutionen betreffen, eignet $\mathrm{da}$ im allgemeinen eine stärkere Verpflich- 
tungsgewalt, resultieren sie doch aus gesetzgeberischen Akten bzw. spiegeln den Willen der wichtigsten gesamtgesellschaftlichen Gruppen, die im nationalen Massenmedium zur Repräsentation drängen und sind nicht etwa bloß Selbstregelungsversuche der publizistischen Institutionen wie im Fall der privat kontrollierten Massenkommunikationsmittel. Während die Codices der letzteren z. B. die Massenbildpresse oder die Filmindustrie gerade vor einer intensiveren staatlichen Normierung bewahren sollen, sind also diejenigen der halbstaatlichen Rundfunk- und Fernsehgesellschaften letztlich Konkretisierungen der bestehenden Rahmengesetzgebung, die durch die wichtigsten gesellschaftlichen Gruppen sanktioniert werden.

Eine Instanz, die das ethische Verhalten der Publizisten in diesen halbstaatlichen Institutionen vorschreiben kann, ist damit zweifellos gegeben, das Problem der publizistischen Ethik indes wird dadurch lediglich auf eine andere Ebene verschoben. Sind nämlich diese Codices eigentliche und detaillierte Gesetzeswerke, dann wird das Problem der publizistischen Ethik einfach durch teilweise Preisgabe des Prinzips der Pressefreiheit gelöst ${ }^{19}$. Ohne eine solche Gesetzgebung vermögen dagegen die proportional den wichtigsten gesellschaftlichen Gruppen besetzten Uberwachungsgremien auch nicht viel mehr als in der publizistischen Produktion Proportionalität gemäß den von ihnen repräsentierten Interessen zu erwirken. Daraus resultieren entweder als publizistische Kardinaltugend die Vorsicht vor diesem „Kartell der stärksten Gruppen “20 und damit eine Ethik des geringsten Widerstandes oder, bei proportionaler Besetzung der publizistischen Schlüsselpositionen solcher Institutionen, ein Nebeneinander von Anhängern verschiedener publizistischer Ethiken und damit eine entsprechend widersprüchliche Produktion, die den geringen Wert solcher Kodifikationen beweist.

Die grundsätzlich geringere Sanktionierbarkeit publizistischer Selbstkontrollen schließlich, zielen sie nun auf private Massenmedien oder auf die publizistischen Berufe, hängt natürlich damit zusammen, daß es sich bei ihnen regelmäßig bloß um interne Absprachen handelt. Ihre Verbindlichkeit endet dort und dann, wo und wenn Massenkommunikationsmittel bzw. Publizisten damit rechnen, ihren wirtschaftlichen, politischen oder kulturellen Zielen ohne oder gegen sie besser dienen zu können. Zumal bei den Selbstkontrollen ganzer Mediengruppen geht es ja viel weniger um ethische Selbstbindung als um Präventivmaßnahmen zur Sicherung eines dadurch zwar beschränkt freien, dafür um so ungestörteren und erfolgreicheren Geschäftsgangs. Die Ethik dieser Vereinbarungen ist somit vornehmlich eine, die das gerade Günstige im Auge hat, und ihre Satzungen werden baldmöglichst revidiert, wenn sich das gesellschaftliche Einflußgefälle verlagert hat.

Auch die Ehrencodices der Publizisten ${ }^{21}$ sind, wenn auch in geringerem Maß, von solcher Doppeldeutigkeit gezeichnet. Bei diesen Regeln, wie z. B. Informationen korrekt $\mathrm{zu}$ sammeln und $\mathrm{zu}$ verwerten seien, handelt es sich ja ebensosehr um öffentliche Beschwichtigungsgesten wie um moralische Binnenzensur, um Standesgeltung und Standespolitik wie um berufliche Leistungskontrolle ${ }^{22}$. Im übrigen hängt natürlich alles davon $a b$, wie weit die publizistischen Institutionen als die Arbeitgeber der Publizisten diese Normen sanktionieren. Je schärfer die Korkurrenz unter diesen ist, desto größer wird offenbar die Wahrscheinlichkeit, daß sie um erfahrungsgemäß zu gewinnender Vorteile willen von ihren publizistischen Mitarbeitern Leistungen verlangen, die nur unter Verletzung dieser ethischen Minimalanforderungen zustandekommen können.

Die häufige Forderung, es dürften Publizisten nicht von ihren Arbeitgebern gezwungen werden, gegen ihre eigene Überzeugung $\mathrm{zu}$ publizieren, spiegelt diese 
Sanktionsproblematik deutlich genug und entspricht gewiß elementaren Normen solcher Gesellschaften. Damit beanspruchen allerdings die Publizisten letztlich doch wieder das Recht, über die Normkonflikte, in die sie bei ihrer Berufstätigkeit geraten, allein zu befinden, und auf diese Weise bleibt das Problem einer zusätzlichen Regelung von Publizistik in Form einer entsprechenden Ethik weiterhin ungelöst.

\section{Publizistische Rollenkonflikte}

Gesamthaft scheinen also die publizistischen Codices eher Alibifunktionen gegenüber der Offentlichkeit zu erfüllen als die publizistische Tätigkeit wirklich gemäß bestimmten ethischen Prinzipien zu steuern. Zugleich kann auch nicht übersehen werden, daß sich die gegenseitige Kritik der Massenmedien in dieser Hinsicht gewöhnlich in sehr bescheidenem Rahmen hält. Darin äußert sich wohl die Soldidarität einer sonst durchaus nicht geeeinten Berufsgattung inkommensurablen gesellschaftlichen Forderungen gegenüber, spiegelt sich ihre Weigerung, die ethischen Widersprüche, welche die Gesellschaft selber hervorbringt, stellvertretend auf sich zu nehmen; im allgemeinen publizistischen Verhalten zeigen sich die ethischen Paradoxien einer von Massenmedien herzustellenden Offentlichkeit überhaupt. Unter den unzähligen ethischen Konflikten, die den Trägern der publizistischen Rollen in solchen Gesellschaften zu lösen angesonnen wird, sollen daher immerhin noch einige grundsätzliche umrissen werden. An ihnen soll zumindest klar werden, was es heißt: Redaktionsdienst zu leisten, zu publizieren, Entscheidungen zu fällen unter widersprïchlichen, ungeklärten ethischen Auflagen.

Als erstes und meistdiskutiertes Dilemma soll dasjenige der Publizisten erörtert werden, die für private, also liberal institutionalisierte Massenkommunikationsmittel tätig sind. Dieser Institutionalisierungsmodus ist dadurch gekennzeichnet, daß, wie erwähnt, einesteils die staatliche Gesetzgebung gewisse Minimalforderungen stellt und andernteils das Massenmedium den wirtschaftlichen Regulierungsmechanismen überantwortet ist. Dem Publizisten wird damit angesonnen, der Gesamtgesellschaft eine für deren Bestand unerläßliche öffentliche Dienstleistung zu erbringen und zugleich die für den Bestand seines Massenmediums unentbehrliche bestverkäufliche Ware zu produzieren. Die Publikumsmaximierung ist folglich ein zwingendes Gebot, da nur dann der betreffenden illustrierten Zeitschrift oder kommerziellen Fernsehstation die Insertionsgelder, auf die sie für ihre Existenz angewiesen sind, in genügendem Maß zuströmen. Genau genommen müssen also diese Publizisten einesteils sogar bloß Massenpublica für Warenverkäufer werben und andernteils ihre Rezipienten mit Informationen versehen, die diesen die Wahrnehmung ihrer demokratischen Partizipationsrechte gestatten. Dazu kommen, als drittes, häufig noch die kulturellen Auflagen der Selbstkontrolle.

Der publizistische Rollenkonflikt gründet nun natürlich darin, daß nach allgemeiner Geschäftserfahrung staatsbürgerlich relevante Information und höhere kulturelle Standards die Publikumsmaximierung im Freizeitraum, in den die Massenmedien ja hineinproduzieren, gewöhnlich erschweren. $\mathrm{Da}$ aber die wirtschaftliche Selbsterhaltung des Massenkommunikationsmittels Voraussetzung ist, daß es überhaupt seinen Beitrag an das Funktionieren der Demokratie und eventuell die kulturelle Wertwelt leisten kann, ist allein schon durch den Institutionalisierungsmodus bis zu einem gewissen Grad der Primat des Wirtschaftlichen gesetzt. Zugleich steht damit solchen Institutionen der Massenkommunikation eine bequeme Ideologie zur Ver- 
fügung, mit der sie ihren Geschäftswillen sowohl vor der Offentlichkeit wie gegen ethisch anspruchsvollere Mitarbeiter rechtfertigen können. Diese hingegen sind offenbar ständig dem Widerspruch zwischen gesinnungsethischen und verantwortungsethischen Verpflichtungen und überdies auch noch demjenigen zwischen verschiedenen Yerantwortlichkeitsbereichen ausgesetzt.

Dem liberalen Institutionalisierungsmodus liegt ursprünglich ein radikal individualistisches Ethos zugrunde, das den Rezipienten zutraut, sie erwählten aus der Fülle des Veröffentlichten das ihnen Zuträgliche, und den Publizisten, ihr individuelles Gutdünken bringe diese Vielfalt zustande. Liberale Institutionalisierung verpflichtet damit den Publizisten zuallererst zu einem gesinnungsethischen Handeln. Das Zeitalter des Liberalismus ist (oder war) nicht zufälligerweise dasjenige der Gesinnungspresse.

Deren Rückläufigkeit beweist indes, daß der größte Teil des Publikums gerade dasjenige nicht mehr will, was viele Postulate der kulturrepräsentierenden und -vermittelnden Gruppen von den Publizisten privater wie halbstaatlicher Massenkommunikationsmittel verlangen, eben die unbedingte Verfechtung bestimmter Normen des Guten, Wahren, Sittlichen. Die Publizisten privater Massenmedien können also aus Verantwortlichkeit für das wirtschaftliche Überleben ihrer publizistischen Institution die in Familie, Schule oder Kirche gelernten ethischen Prinzipien nicht kompromißlos praktizieren, und die Mitarbeiter halbstaatlicher Massenkommunikationsmittel nicht, weil diesen Monopolbetrieben die Repräsentation unterschiedlichwidersprüchlicher Kulturnormen obliegt, und auch aus Verantwortung für das nur von Fall zu Fall bestimmbare Gemeinwohl. Darum werden gerade von ihnen z. B. Aufwertungspläne der Regierung noch entschieden dementiert, wenn Indiskretionen von Aufwertungsgegnern sie längst vom Gegenteil überzeugt haben.

Im Problem der publizistischen Ethik offenbart sich damit wieder dasjenige des öffentlichen, stellvertretenden Handelns überhaupt ${ }^{23}$. Geplante Veränderungen der Währungsparität z. B. werden ja auch von den Regierungen regelmäßjg unter systematischer Irreführung der Offentlichkeit verwirklicht, um des wirtschaftlichen Gemeinwohls willen. In situationsethischer Entscheidung wird auch von ihnen immer wieder praktiziert, was Merton Kate Smith vorwarf: die Manipulation der Allgemeinheit im Interesse der Allgemeinheit. Während kulturrepräsentierende und -vermittelnde Gruppen ein Stellvertretungsrecht bei ihren prinzipiellen Postulaten beanspruchen, das ihnen nicht zusteht, verraten also die legitimen Stellvertreter der Allgemeinheit von Fall zu Fall die Prinzipien, unter die sie - zumindest öffentlich - ihr Handeln stellen ${ }^{24}$. Prinzipielles fordern die ersteren, ohne daß sie dafür die praktische Verantwortung tragen müssen; Verantwortung wird, im günstigen Fall, von den letzteren bewiesen, und mit einem späteren Wahlerfolg sanktioniert vielleicht die Gesamtgesellschaft als die einzige designierbare Instanz ihr Verhalten.

Von den Publizisten hingegen wird in solchen Gesellschaften offenbar beides erwartet: Gesinnungs- und Verantwortungsethik zugleich, und die letztere soll wiederum so beschaffen sein, daß sie das Plebiszit des Publikums gewinnt, aber auch das Gemeinwohl verbürgt. Zwar wird im 20. Jahrhundert vor allem der Ruf nach einer publizistischen Verantwortungsethik laut 25 , diese allein genügt indes nicht, um z. B. den Pragmatismus der Regierenden zu kontrollieren ${ }^{26}$. Neben dem jüngeren halbstaatlichen Institutionalisierungsmodus, einem demokratischen Regelungstyp, der bis zu einem gewissen Grad publizistische Verantwortungsethik erzwingt, besteht denn auch nach dem Willen dieser Gesellschaften die liberale, die private Institutionalisierungsweise von Massenkommunikationsmitteln weiter. 
Manipulation der Allgemeinheit im Interesse der Allgemeinheit scheint freilich in solchen demokratischen Großgesellschaften, wo über eine durch Massenmedien hergestellte Offentlichkeit das allgemeine Gute verwirklicht werden muß, das weitaus tauglichste Mittel zu sein, dieses Ziel zu erreichen. Das jeweilige Gemeinwohl, wenn es von den kompetenten und verantwortlichen Stellen ermittelt worden ist, ist ja meist dermaßen kompliziert, das Resultat von Spezialistenkalkulationen im Bereich der sekundären Systeme27, daß es das Durchschnittsverständnis und die allgemeine praktische Lebenserfahrung bei weitem übersteigt. Darum gehört bekanntlich zur demokratischen Regierungstechnik vor allem auch die Kunst des „Wie sag ich's meinen Kindern", und zwar im wörtlichsten Sinn: Zustimmung zum Notwendigen im entfremdeten sekundären Sozialhorizont wird nämlich regelmäßig durch den Appell an moralische Vorstellungen gewonnen, die primärgruppenhaften Verpflichtungen entstammen und entsprechen, also im Grunde durch die psychologische Ausbeutung des primären Sozialhorizonts ${ }^{28}$. Es bringt eben die demokratische Großgesellschaft keine umfassend-verbindliche Ethik hervor, die ihren Pluralismus und ihre Dichotomien unter anspruchsvolleren Kulturnormen wieder vereinigte, sondern nur eine Offentlichkeitstechnik, die von Fall zu Fall irgendein gesellschaftliches Funktionieren ermöglicht, und ihrer bedienen sich wie die Regierenden, die Geschäftsleute und die politisierenden Kirchen auch die Publizisten.

Der Großerfolg von Kate Smith und ihrer publizistischen Ratgeber bei der Sicherung der amerikanischen Währung verdeutlicht damit noch einmal unmißverständlich das ganze Problem: Es gibt in solchen Gesellschaften eine wirksame publizistische Technik des Vermittelns, aber keine verbindliche publizistische Ethik des Uhersetzens. $\mathrm{Zu}$ übersetzen sind $\mathrm{ja}$ von allen stellvertretend und öffentlich Handelnden gesellschaftliche Tatbestände und Traktanden in öffentliche Aussagen, auf Grund derer die Gesellschaftsmitglieder ihre Entscheidungen fällen können und sollen. Sach- und publikumsgerecht müßte somit Publizistik als das wichtigste Medium dieser Ubersetzung sein, authentischer Ersatz für die fehlende Direkterfahrung einer weiteren Umwelt, die einen bestimmt und über die man befinden muß, obwohl man sie nicht durchschaut. Weil aber die Rezeptionsvorgänge gruppenhaft und subjektiv verschieden verlaufen, läßt sich auch Sachgerechtigkeit, „Objektivität ${ }^{\star}$, nicht allgemein gültig bestimmen. Entsprechenden Normierungen eignet darum regelmäßig die Willkür der gerade gegebenen Machtverhältnisse, wie extrem im totalitären Institutionalisierungsfall, oder das geringe materiale Bestimmungsvermögen bloßer allgemeiner Sicherungstechniken, wie in den erwähnten publizistischen Ehrencodices.

Der publizistische Schein in solchen Gesellschaften, damit er doch das Vertrauen der Rezipienten erhält, muß deshalb zumindest authentisch wirken, und in dieser Richtung verbessern sich denn auch konsequenterweise die technischen Apparate und professionellen Leistungen weitaus am stärksten ${ }^{29}$. Auch schon an Kate Smith war es vor allem ihre "Echtheit", welche die Zuhörer überzeugte und sie zu Opfern gemäß einer Moral trieb, auf welcher der Unterhaltungsstar lediglich virtuos spielte, zu Opfern, die indes gesamthaft auch wieder im wohlverstandenen Interesse dieser Rezipienten lagen und überdies Forderungen abstrakterer und von diesen gleichfalls anerkannter Normen erfüllten. So stark ist das Authentizitätserlebnis, das die Publizisten, nicht zuletzt um auch Gutes zu erreichen, ihren Rezipienten verschaffen, daß der Glaube an die Naturwahrheit des publizistischen Fotos und an die Televisionskamera als Lügendetektor nicht ausstirbt. Immer wieder wird aber auch solch gutgemeinte Publizistik als Schein, als bloße Veranstaltung 
entlarvt, und dann verstärkt sich das latente Mißtrauen gegen die unberechenbar fremde Umwelt, die doch die eigene Gesellschaft ist, oder wird in individuellen und kollektiven Globalverdächtigungen von Publizistik als ständiger Manipulation manifest ${ }^{30}$.

Bei der Frage nach der publizistischen Ethik offenbart sich damit noch einmal eine verantwortungsethische Verpflichtung der öffentlich und stellvertretend Handelnden und Redenden schlechthin, nämlich diejenige zum gemeinwohlgerechten Abwägen der funktionalen und dysfunktionalen Wirkungen ihres Tuns. Mit wachsender Enttäuschung der Rezipienten verlieren ja z. B. nicht nur die Massenkommunikationsmittel die von Merton beschriebenen Mobilisierungskräfte zum gemeinsamen Guten, sondern zerbröckelt auch unter kurzfristigen Erfolgen das politische Fundament der Demokratie: ein gewisses Vertrauen in die öffentlich Tätigen ${ }^{31}$.

Umgekehrt könnte aber voraussichtlich auch wache Publikumskritik die Publizisten allmählich auf höhere ethische Standards als die jetzt gemeinhin von ihnen eingehaItenen verpflichten. Das Publikum indes, begeistert, daß es überhaupt vor der Kamera posieren darf oder in die Gazetten kommt, spielt selbst immer wieder mitt $^{32}$, wenn es um die Herstellung jenes schönen oder erregenden Scheins von Authentizität geht, den Publizistik offenbar zu geben hat und den man ihr zugleich vorwirft. Kritik und Komplizenschaft vermengen sich so auf Seiten des Publikums aufs unentwirrbarste, und darum hält es sogar die Verwendung gestellter Fotos als angeblich authentischer publizistischer Situationszeugnisse nur in krassen Fällen für unstatthaft ${ }^{33}$. Ein Gutteil der Vorwürfe, die das Publikum gegen die Publizisten erhebt, fällt damit auf dieses selbst zurück.

Der gern und oft geführte Schuldbeweis der Publizisten gegen das Publikum ist damit freilich so wenig geleistet wie derjenige der postulierenden Gruppen gegen die Publizisten. Als weitere, letzte Frage im Zusammenhang mit dem Problem der publizistischen Ethik drängt sich ja hier sofort auf: Kann das Publikum überhaupt wollen ${ }^{34}$, wenn es von der Publizistik anders konditioniert wird? Warum aber, stellt sich gleich die Gegenfrage ein, warum, wenn dem so ist, sind solche Gesellschaften so beschaffen, daß das Publikum sich auf diese Weise von der Publizistik konditionieren läßt? Auf diese Wechselfragen sind offenbar, ihrer Allgemeinheit wegen, keine sinnvollen Antworten mehr möglich. Immerhin weisen sie in einem letzten Horizont noch einmal auf einen Aspekt der Dialektik von Publizistik und demokratischen Gesellschaften hin, die das Problem der publizistischen Ethik nicht zur Lösung kommen läßt: Weil das Publikum nicht will, müssen die Publizisten nicht; weil die Publizisten nicht müssen, kann das Publikum nicht wollen.

\section{Anmerkungen:}

1. Robert K. Merton: Mass Persuasion, New York-London 1946.

2. Merton: a.a.O., S. 175-189.

3. Merton: a.a.O., S. $187 / 188$.

4. Vgl. z. B. Klaus Lefringhausen: Ethik des Informierens, in: „Die Mitarbeit. Zeitschrift zur Gesellschafts- und Kulturpolitik“, 15. Jg. (Mai/Juni 1966), S. 235 f.

5. Vgl. z. B. Renate Mayntz: Soziologie in der Eremitage?, in: Logik der Sozialwissenschaften, hrsgg. v. Ernst Topitsch, Köln-Berlin 1965, S. 526. Bezeichnend ist auch etwa, daß seit den 30er Jahren die Beiträge zu Themen der publizistischen Ethik im „Journalism Quarterly " immer seltener werden.

6. Vgl. Soziologie, hrsgg. v. René König, Frankfurt a. M. - Hamburg 1967, S. 102, 298 und 322; zur Notwendigkeit eines umfassenden Betrachtungsansatzes siehe auch R. S. Downie: Government Action and Morality, London 1964, S. 15; Theodore Peterson, Jay W. Jensen und William L. Rivers: The Mass Media and Modern Society, New York/Chicago/San Francisco/Toronto/London 1965, S. 245. 
7. Vgl. Judith Janoska-Bendl: Methodologische Aspekte des Idealtypus, Berlin 1965.

8. Vgl. zu den totalitären Gegebenheiten die klassische Darstellung von Alex Inkeles: Public Opinion in Soviet Russia, Cambridge, Mass. 1950.

9. Vgl. z. B. E. J. B. Rose: Problems of Professional Standards, in: Press Councils and Press Codes, hrsgg. v. The International Press Institute, Zürich 41966, S. 3.; Jay W. Jensen: Freiheit der Presse: Ein Begriff auf der Suche nach einer Philosophie, in "Publizistik“, 12. Jg. (1967), H. 2/3, S. 103.

10. Vgl. A Dictionary of the Social Sciences, hrsgg. v. Julius Gould und William L. Kolb, New York 1964, S. 29/30; Merton: a.a.O., S. 10/11.

11. Max Weber: Politik als Beruf, in: Gesammelte politische Schriften, hrsgg. v. Johannes Winckelmann, Tübingen ${ }^{2} 1958$, S. $536 \mathrm{f}$. Freilich sollen die beiden Ethiken hier doch nicht dermaßen absolut einander entgegengesetzt werden, wie dies Max Weber tut (Vgl. Raymond Aron: Max Weber und die Machtpolitik, in: Max Weber und die Soziologie heute, Verhandlungen des 15. deutschen Soziologentages, Tübingen 1965, S. 117-119). Aufschlußreiche Beispiele gesinnungs- bzw. verantwortungsethischer Haltung in der heutigen Gesellschaft zitiert und diskutiert Gerhard Schmidtchen: Die befragte Nation, Frankfurt a. M. - Hamburg 1965, allenthalben.

12. Zum Begriff "Pluralismus" vgl. auch Staat und Politik, hrsgg. v. Ernst Fraenkel und Karl Dietrich Bracher, Frankfurt a. M. - Hamburg ${ }^{2} 1964$, S. 254.

13. Vgl. Helmut Schelsky: Gedanken zur Rolle der Publizistik in der modernen Gesellschaft, in: Schelsky: Auf der Suche nach Realität, Düsseldorf-Köln 1965, S. 310 f.

14. Solche Codices enthalten und diskutieren u. a. Stefan Bamberger: Christentum und Film, Aschaffenburg 1968; Die pädagogische Chance der technischen Medien, hrsgg. v. Heinrich Berresheim und Herbert Hoersch, Düsseldorf 1964; Karl-Werner Bühler: Die Kirchen und die Massenmedien, Hamburg 1968; Press Councils and Press Codes, hrsgg. v. The International Press Institute, a.a.O.; Selbstkontrolle von Presse, Funk und Film, hrgg. v. Martin Löffler, München-Berlin 1960; William L. Rivers: The Mass Media, New York 1964; Presse-Reform und Fernseh-Streit, hrsgg. v. Otto B. Roegele und Peter Glotz, Gütersloh 1965; Wilbur Schramm: Responsibility in Mass Communication, New York 1957; Mass Media and Communication, hrsgg. v. Charles S. Steinberg, New York 1966.

15. Vgl. z. B. Karl-Werner Bühler: a.a.O., S. 81/82; Press Councils and Press Codes, a.a.O., allenthalben; John C. Merrill: Who Can - or Should - Evaluate the Press?, in: "Gazette“, Vol. XII (1966), No. 2/3, S. 169.

16. Vgl. Anm. 14; ferner insbesondere Gustav Ermecke: Pressefreiheit und Presseverantwortung, Paderborn 1965, allenthalben; Wilbur Schramm: a.a.O., S. 292 ff.; Eberhard Stammler: Die erzieherische Seite der Selbstkontrolle, in: Selbstkontrolle von Presse, Funk und Film, hrsgg. v. Martin Löffler, a.a.O., S. 26-29; Fernand Terrou und Lucien Solal: Le droit de l'information, Paris 1951, S. $413 \mathrm{f}$.

17. Vgl. u. a. Theo Fürstenau: Form und Moral, in: „Publizistik“, 5. Jg. (1960), H. 6, S. 399-408; Mass Media and Communication, hrsgg. v. Charles S. Steinberg, a.a.O., S. $493-517$.

18. Vgl. z. B. Wilbur Schramm: a.a.O., S. 103/104; Eberhard Stammler: IllustriertenSelbstkontrolle vor einer Wende, in: „ZV+ZV $\mathrm{Z}^{\alpha}$, 65. Jg. (1968), H. 25, S. $1070 \mathrm{f}$.

19. Vgl. z. B. Press Councils and Press Codes, a.a.O., S. 71.

20. Claus-Peter Gerber und Manfred Stosberg: Die Massenmedien und die Organisation politischer Interessen, Bielefeld 1969, S. 41.

21. Press Councils and Press Codes, a.a.O., allenthalben.

22. Vgl. u. a. Press Councils and Press Codes, a.a.O., S. 60; Form und Funktion der Presse-Selbstkontrolle, hrsgg. v. Martin Löffler und Jean Louis Hébarre, München 1968, S. $7 \mathrm{f}$;; G. Mueller: Zensur und Selbstkontrolle der Massenmedien in den Vereinigten Staaten von Amerika, in: Selbstkontrolle von Presse, Funk und Film, hrsgg. v. Martin Löffler, a.a.O., S. 30-38; Gerhard Schmidtchen: a.a.O., S. 151; Edgar Stern-Rubarth: Ethik und Geschäft der Presse, in: „Publizistik“, 9. Jg. (1964), H. 3. S. 209-212.

23. Den ganzen Fragenkomplex diskutiert sehr instruktiv $R$. S. Downie: a.a.O.

24. Vgl. z. B. Maurice Duverger: Introduction à la politique, Paris 1964, S. 249/250.

25. Vgl. u. a. The Commission on Freedom of the Press: A Free and Responsible Press, Chicago 1947; Wilbur Schramm: a.a.O.

26. Vgl. Ludwig Freund: Politik und Ethik, Gütersloh 21961, S. 299.

27. Hans Freyer: Theorie des gegenwärtigen Zeitalters, Stuttgart 1955, S. $81 \mathrm{f}$.

28. Vgl. z. B. Merton: a.a.O., allenthalben. 
29. Von der Presse über den Hörfunk bis zum Fernsehen wird ja den Rezipienten ein ständig zunehmendes Gefühl des "Dabei-Seins“ geboten.

30. Vgl. z.B. die stark gesinnungsethisch motivierten international zu beobachtenden Jugendrevolten.

31. Vgl. u. a. Rudolf Wildenmann und Werner Kaltefleiter: Funktionen der Massenmedien, Frankfurt a. M. - Bonn 1965, S. 62.

32. Vgl. u. a. Kurt Lang und Gladys Engel Lang: The Unique Perspective of Television and its Effects: A Pilot Study, in: Mass Communications, hrsgg. v. Wilbur Schramm, Urbana, III. ${ }^{2} 1960$, S. 557.

33. Walter Wilcox: The Staged News Photograph and Professional Ethics, in: „Journalism Quarterly“, vol. 38 (autumn 1961), No. 4, S. 497 f.

34. Theodor W. Adorno: Kann das Publikum wollen? in: Vierzehn Mutmassungen über das Fernsehen, hrsgg. v. Anne Rose Katz, München 1963, S. 55-60.

\section{SUM M A R Y}

Particular ethics for mass media work are requested especially in democratic industrial societies. Following the principle of the freedom of the press, the mass media stands outside the ruling of governmental machinery. This is because mass media are responsible to society in a larger sense. The pluralism of these societies, on the other hand, makes it impossible to create ethics for mass media work in a way agreeable to everybody. The mass media ethics, therefore, are somehow self-contradictory. They are making very often mutual allowances to the postulates of these groups and serve the journalists as an alibi. The moral power of these semi-spontaneous attempts at self-regulation are anyhow weak and the many conflicts which arise very often are due to the conflicts between the duty for economic self-existence and the duty to the general public. A main problem of mass media ethics is furthermore the question of how far a manipulation of the general public is allowed in the interest of this very same general public.

\section{RESUMEN}

Hoy día se exige una ética periodística especial, sobre todo en las sociedades democráticas industriales. Siguiendo el principio de la libertad de prensa, los medios de comunicación se hallan fuera de la maquinaria jurídica gubernamental. Sucede esto porque los medios de comunicación son responsables ante la sociedad en general. Por otra parte, el pluralismo de estas sociedades imposibilita el establecimiento de una ética del trabajo de comunicación de masas que sea válida para todos. - Así, pues, la ética de los medios de comunicación social es, en cierto modo, auto-contradictoria. Frecuentemente se hacen concesiones a los postulados de los diversos grupos, las cuales son utilizadas por los periodistas como alibís. El poder moral de estos semi-espontáneos tentativos de autocontrol y los numerosos conflictos que muchas veces surgen son debidos al choque entre los deberes de automantenimiento económico y el de prestar un servicio responsable a la sociedad. Otro grave problema de la ética de los medios de comunicación social es la cuestión de si el lícita la manipulación de la sociedad en general en beneficio de la misma. 